\begin{tabular}{|l|l|}
\hline & HHS PUblic ACCESS \\
$\begin{array}{ll}\text { Author manuscript } \\
\text { Curr Opin Biomed Eng. Author manuscript; available in PMC } 2019 \text { September } 01 .\end{array}$ \\
\hline
\end{tabular}

Published in final edited form as:

Curr Opin Biomed Eng. 2018 September ; 7: 42-50. doi:10.1016/j.cobme.2018.09.005.

\title{
Polymeric Nucleic Acid Delivery for Immunoengineering
}

\author{
Stephany Y. Tzenga,b and Jordan J. Green ${ }^{a, b, c, d, e,}{ }^{*}$ \\ aDepartment of Biomedical Engineering and the Translational Tissue Engineering Center, Johns \\ Hopkins University School of Medicine, Baltimore, MD 21231, USA \\ ' Institute for Nanobiotechnology, Johns Hopkins University, Baltimore, MD 21218, USA \\ 'Departments of Materials Science and Engineering and Chemical and Biomolecular \\ Engineering, Johns Hopkins University, Baltimore, MD 21218, USA \\ dDepartments of Oncology, Ophthalmology, and Neurosurgery, Johns Hopkins University School \\ of Medicine, Baltimore, MD 21231, USA \\ eBloomberg Kimmel Institute for Cancer Immunotherapy, Johns Hopkins University School of \\ Medicine, Baltimore, MD 21231, USA
}

\begin{abstract}
Nucleic acids hold promise as tools for engineering the immune system. However, for use in patients, their delivery must be improved to overcome challenges in specificity and intracellular delivery efficiency. Polymers, both natural and synthetic, are under investigation as nucleic acid delivery vehicles, and their chemical and physical properties are being engineered to enhance their efficacy. Through formulation with polymers, DNA, mRNA, siRNA, and other nucleic acids can serve as prophylactic or therapeutic vaccines by overexpressing or knocking down expression of genes that affect the immune response. Polymeric delivery is also useful for the transport of intrinsically immunostimulatory nucleic acids to specific compartments of cells. Recent innovative examples of polymer-based nucleic acid vaccines and immunomodulatory strategies will be discussed.
\end{abstract}

\section{Graphic Abstract}

*Correspondence: green@jhu.edu, 400 N Broadway, Smith Bldg, Room 5017, Baltimore, MD 21231, USA.

The authors declare no conflict of interest.

Publisher's Disclaimer: This is a PDF file of an unedited manuscript that has been accepted for publication. As a service to our customers we are providing this early version of the manuscript. The manuscript will undergo copyediting, typesetting, and review of the resulting proof before it is published in its final citable form. Please note that during the production process errors may be discovered which could affect the content, and all legal disclaimers that apply to the journal pertain. 


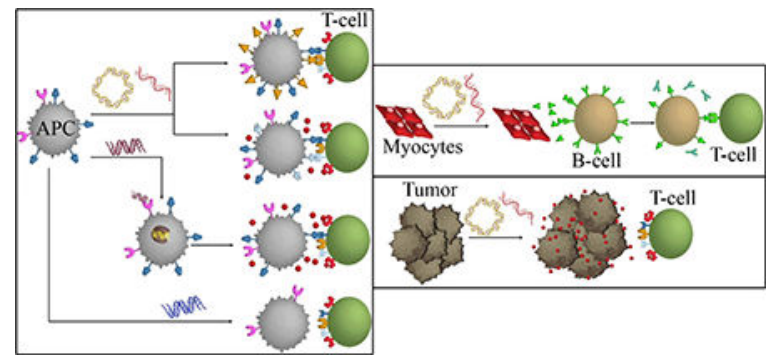

\section{Introduction}

The role of the immune system in various aspects of health and disease has become increasingly delineated over the past decades. In tandem, biomedical engineers have been putting increased emphasis on the discovery and development of new technologies to harness or alter the immune response in order to treat or prevent disease. Among the emerging immunoengineering technologies is the intracellular delivery of nucleic acids, including DNA and many types of RNA. Although historically achievable using viruses, the use of live viruses has several drawbacks, including technical manufacturing challenges, limited cargo capacity, unintended side effects, and the inherent safety and immunological properties of viruses, all of which have led to the development of non-viral vehicles for nucleic acid delivery. This review will describe the use of polymers as a non-viral method for delivering nucleic acids for immunoengineering purposes, with emphasis on strategies and technologies that have arisen in recent years.

\section{Nucleic Acids as Cargo}

There are many important types of immunostimulatory nucleic acids that can act as genetically encoded programming messages or directly as adjuvants to more strongly activate immune cells, including plasmid DNA, mRNA, and siRNA (Figure 1). A major delivery challenge for nucleic acid types that are active within the cell is that they are large, hydrophilic and highly negatively charged molecules, all of which hinder their ability to pass through cell membranes unaided. Plasmid DNA, which is double-stranded, circular, and generally $>2 \mathrm{kbp}$ in size, must overcome multiple barriers [1]. First, it must be internalized into cells; then, in order to avoid degradation or recycling through the endosomal/lysosomal pathway, the DNA must escape the endosome. Once out of the endosome and in the cytoplasm, the DNA must travel to and pass through the nuclear membrane. Only when it is in the nucleus does the DNA have a chance to be expressed and transcribed into mRNA.

mRNA and siRNA face some of these same challenges but differ in key ways [2,3]. RNA is in less chemically stable than DNA. Single-stranded mRNA is easily degraded, both outside and within the body; however, mRNA is translated into protein in the cytosol, which eliminates the challenge of nuclear localization and entry faced by plasmid DNA. Moreover, because DNA enters the nucleus and can potentially recombine with the genomic DNA of the cell, there is a small but extant risk of its uncontrolled integration into the genome. mRNA does not carry this risk, but because it is transcribed from DNA and traditionally does not self-amplify, it is also more challenging to manufacture stably in large quantities. 
siRNA, a short ( $20 \mathrm{kbp})$ doublestranded molecule, is also active in the cytosol and, like mRNA, needs to be released before reaching the nucleus. However, while mRNA and plasmid DNA can both be used to overexpress genes, siRNA leads instead to knockdown or reduced expression of a specific gene, using the RNA interference (RNAi) pathway to block the translation of a target mRNA sequence or effect targeted mRNA degradation.

Finally, some nucleic acids have inherent immunostimulatory properties, often because they resemble foreign nucleic acids from pathogens and activate certain receptors on immune cells. These nucleic acids include DNA with regions that contain unmethylated cytosine followed by guanine (CpG) [4] and immunostimulatory RNA (isRNA) [5], which can function for immunostimulation within the endosome due to interactions with Toll-like receptors (TLR) and therefore do not need to escape to the cytosol [6]. Other immunostimulatory nucleic acids can activate an innate immune response, including cyclic dinucleotides (CDN) [7], which activate STING and lead to a Type I interferon response. Thus, nucleic acids can stimulate the immune system against a pathogen or can be developed into an innovative cancer vaccine, but they require a delivery system.

\section{Polymers for Nucleic Acid Delivery}

A wide range of polymers has been developed for nucleic acid delivery (Figure 2). A prominent feature of most of these is their cationic nature. Because nucleic acids are highly negative, positively charged polymers can easily complex with them, aiding in the condensation of the normally large cargo and often self-assembling it into nanoparticles of approximately $50200 \mathrm{~nm}$, a diameter that makes nanocomplexes amenable to cellular uptake. Many early gene delivery studies used poly(L-lysine) (PLL), which efficiently bound to the cargo but was limited by the inability to facilitate endosomal escape and cargo release inside the cell as well as its cytotoxicity [8]. Reversibly protonated polymers like polyethylenimine (PEI), due to their buffering capacity, can better overcome intracellular barriers like endosomal escape [9], but while PEI and variations on its basic structure continue to be used for gene delivery, it is limited by toxicity, being a large, charged, nondegradable polymer [10].

In an effort to increase safety, multiple research groups have developed degradable cationic polymers, which retain the advantageous cationic and buffering properties while being less toxic. These include poly( $\beta$-amino ester)s (PBAEs) [11], which degrade by hydrolysis. The use of synthetic polymers allows the introduction of various chemical or biochemical groups that can degrade by hydrolysis or in response to a desired stimulus, such as an increase in protease activity or changes in $\mathrm{pH}$ or redox potential, like disulfide-containing poly(amido amine)s (PAMAMs) [12]. This leads not only to low toxicity but also to the potential of targeted delivery of nucleic acids to particular tissue types or intracellular compartments. For instance, reducible polymers may be advantageous for delivery of mRNA and siRNA to the cytosol, as the cytosol of cells has approximately three orders of magnitude higher concentration of the reducing agent glutathione than the extracellular environment [13].

Synthetic PAMAMs are more frequently used as dendrimers, which have a high amine density and a branching structure, both of which help it to complex with and condense 
negatively charged nucleic acids [14]. Like PEI, PAMAM dendrimers suffer some drawbacks, including the low efficacy of low molecular weight dendrimers and the high toxicity of the more effective high molecular weight versions. Due to the high degree of synthetic control over the chemistry of dendrimers, many types of dendrimers exist and have been modified for nucleic acid delivery, including versions with degradable linkages [15].

Chitosan is derived from a natural polysaccharide, and, as a cationic polymer with low toxicity, unlike most of the others discussed so far, it has also been employed for nucleic acid delivery [16]. However, chitosan also has some limitations. It is poorly soluble in water at neutral $\mathrm{pH}$ and thus cannot be processed for use at high dosages without the use of acid solvents. In addition, without modification, its transfection efficacy is moderate in comparison to other common types of polymers.

Finally, as an alternative to electrostatic complexation with cationic polymers, nucleic acids have also been encapsulated into solid polymer matrices. In these systems, while chargecharge interactions between the cargo and the polymer may play a role in determining properties like loading efficiency and release kinetics, the mechanism for nucleic acid encapsulation is largely through physical entrapment. Poly(lactic-co-glycolic acid) (PLGA), related polyesters like poly(lactic acid) (PLA), and other hydrolytically degradable hydrophobic polymers like poly(ortho ester)s (POEs) have been used extensively in the field of drug delivery in general. These types of polymers can form emulsions or mixtures with hydrophilic cargo like nucleic acids, and a precipitation or solvent extraction method is used to solidify the polymer around the cargo, trapping it inside micro- or nanoparticles. Their long history of usage, ease of fabrication, and versatility make them common vehicles of choice, but they are limited by a lack of nucleic acid stability after encapsulation [17] as well as poor encapsulation efficiency unless other materials, such as emulsifiers, are used. One promising approach is the combination of materials generally regarded as safe, like PLGA, with other materials, such as polyamines, to improve encapsulation and intracellular delivery of nucleic acids [18].

It should be noted that all of the broad classes of polymers described here can be chemically modified, such as with the conjugation of T-cell specific ligands for more efficient and targeted uptake [19] or the introduction of degradable functional groups to reduce toxicity [20]. Multiple types of polymers can also be combined to draw on the advantages of different materials into a hybrid delivery system. Continual work on these polymers is being conducted to improve their properties for nucleic acid delivery.

\section{Immune Cell Targets}

Several major players of the immune system are of interest as targets of nucleic acid delivery. Antigen-presenting cells (APCs), particularly dendritic cells (DCs) as well as macrophages and B-cells, are specialized for internalization of antigens from the environment, which they can then process into peptides that are loaded into major histocompatibility complex (MHC) molecules and displayed on the surface [21]. The additional presence of danger signals, or adjuvants, can induce maturation of APCs and greater antigen processing efficiency. The MHC:antigen, often called "signal 1," is presented 
to T-cells, where it binding to a T-cell receptor (TCR) and determines the specific antigen that the T-cell will then recognize [22].

A second interaction or combination of interactions, called "signal 2" or co-stimulation, determines the action that the T-cell will undertake upon recognition of the antigen. Costimulatory molecules on the APC surface, such as B7.1/2 (CD80/CD86), induce T-cell activation by binding with CD28, while B7.1/2 binding to CTLA-4 leads instead to downregulation of T-cell responses, resulting in some cases in T-cell anergy or apoptosis. Other soluble signals ("signal 3") produced by APCs, such as cytokines, also direct the recruitment, expansion, and phenotype of T-cells, and thus the coordination of these various signals is crucial to achieving the desired immune response [22,23]. APCs, which present signals 1-3 to T-cells, are therefore an attractive target for genetic engineering as a means to direct the immune response.

Depending on the nature of the antigen and other signals, several broad types of T-cells can be generated. Cytotoxic T-cells are $\mathrm{CD} 8^{+}$and recognize the degradation products of cytosolic antigens, which are loaded onto MHC class I molecules. Activated cytotoxic CD8 ${ }^{+}$ T-cells are specialized to cause apoptosis in cells that have been infected or become malignant. Thus, MHC class I is expressed not only on professional APCs but also on other cells in the body. $\mathrm{CD} 4^{+} \mathrm{T}$-cells, known as helper cells, recognize peptide fragments derived from extracellular antigens that are presented on MHC class II molecules, expressed largely on the professional APCs and on some other tissue in the body [24]. These T-cells release factors to modulate the effects of other immune cells, including $\mathrm{CD} 8^{+} \mathrm{T}$-cells and B-cells. A subset of $\mathrm{CD}^{+}{ }^{+} \mathrm{T}$-cells are known as regulatory T-cells or Tregs, and can suppress the function of or even cause apoptosis in effector T-cells. While Tregs are essential to prevent autoimmunity, their presence can be problematic in certain contexts in which an active immune response is desirable, including cancer [25]. Generation of long-lived memory Tcells allow robust and quick anamnestic responses upon later reintroduction of an antigen, leading to long-lasting immunity [26]. Finally, B-cells recognize extracellular antigens via the B-cell receptor (BCR) and present antigen peptides on MHC class II molecules. Upon activation by helper T-cells, activated Bcells, or plasma cells, proliferate and secrete antigenspecific antibodies.

\section{Prophylactic Vaccines}

Prophylactic vaccines against infectious diseases are considered among the most lifesaving innovations in modern medicine [27] and are an early example of immunoengineering. While traditional vaccines use a protein antigen or an attenuated form of the pathogen, progress has been made in the field of DNA and mRNA vaccines delivered via polymers, which is attractive due to the low cost of manufacturing nucleic acids and their relative stability. In addition, injection of DNA or mRNA, such as into muscle, can result in either antigen secretion by cells like myocytes, leading to a humoral (antibody-based $\mathrm{CD} 4^{+}$) response, or direct expression by APCs, leading to a cell-mediated (cytotoxic CD8 ${ }^{+}$) response, the latter of which is important for diseases like HIV that require a $\mathrm{CD} 8^{+}$cytotoxic response for immunity and which is difficult to generate via soluble protein antigens [28]. 
However, aside from the challenges associated with intracellular delivery of DNA, DNA vaccines also tend to have poor immunogenicity [29].

Initial work in polymer-based genetic vaccines used DNA encapsulated in microspheres, taking advantage of the ability of DCs to take up micron-sized particles made of PLA, PLGA, chitosan, and/or PEI [30,31]. The DCs then express the DNA and upregulate maturation markers or show increased protection after immunization in vitro and ex vivo [32]. Many groups have recently investigated the use of microneedle patches to deliver DNA to the skin, targeting the DNA intradermally to a site rich with immune cells in order to improve immunogenicity. DNA complexed with a cationic, mannose-based polymer was coated onto the surface of microneedles, and application to mouse skin led to a strong antibody response against the delivered antigen $\alpha \beta$ (Fig. 3) [33]. Another group directly addressed the problem of low DNA immunogenicity by incorporating a synthetic analogue for double-stranded RNA, poly(I:C), as an adjuvant. Here, DNA and poly(I:C) were electrostatically loaded onto microneedles between layers of cationic PBAE, and immunized mice generated a strong CD4 and CD8 immune response against an HIV antigen [34]. The delivery of nucleic acid adjuvants, such as poly(I:C) and CpD ODN, along with an antigen to enhance the immunogenicity of a vaccine has been investigated extensively and continues to be an area of active study [35,36].

\section{Therapeutic Immunoengineering}

Delivery of nucleic acids can be used for therapeutic as well as prophylactic purposes, which leads to applications in diseases that must be combatted with a cell-mediated immune response, including cancers, influenza, and HIV. Because tumor cells themselves are poor APCs, another way to generate a $\mathrm{CD} 8^{+} \mathrm{T}$-cell cytotoxic response is to transfect DCs and other APCs to express the antigen of interest, which can result in MHC class I presentation of the tumor antigen. This can be done ex vivo by isolating patient DCs, transfecting them in vitro, and reintroducing them into the patient by adoptive transfer [37]. Ex vivo manipulation of DCs can be advantageous in that APC maturation can take place under welldefined conditions, but the labor, cost, and challenges to the patient required by this type of procedure makes it unlikely to be broadly applied to cancer patients. In situ transfection of APCs, while potentially a cheaper and off-the-shelf strategy of immunotherapy, carries its own challenges as well. The DNA must be targeted or trafficked to APCs, and transfection efficiency by non-viral methods is generally much lower in this context.

A different strategy that has seen success in recent years is the use of polymers to deliver immunostimulatory nucleic acids to APCs. These nucleic acids act as adjuvants, and rather than encoding an antigen, they promote activation and maturation of APCs, leading to more efficient signaling by APCs and a more immunostimulatory environment. Cyclic dinucleotides (CDNs), for example, bind to stimulator of interferon genes (STING) in the cytosol of APCs. There, they induce expression of type I interferons (IFNs), which in turn signal to other immune cells and have been shown to be effective in causing T-cell-mediated tumor rejection [38]. However, CDNs are limited by their poor cellular uptake, like other nucleic acids. Our group has recently shown that cationic PBAEs can form complexes with CDNs that promote uptake by APCs and lead to significantly reduced tumor growth after 
injection in vivo [39]. Others have encapsulated CDNs in hydrogels for a similar purpose, using the release kinetics from the hydrogel to extend CDN delivery to the tumor site, and showed significantly slower tumor growth and drastically improved survival [40]. Other immunostimulatory nucleic acids can also be used to enhance an immune response, as in the case of the Toll-like receptor (TLR) ligand CpG oligodeoxynucleotides (ODN), co-delivered with an antigen and short hairpin RNA (shRNA) [41]. The cationic polypeptide- $g$-PEG vehicle compacted the CpG ODN and shRNA nucleic acids into what the authors termed intertwining DNA-RNA nanocapsules (iDR-NCs) that trafficked to draining lymph nodes and increased the maturation of APCs, as well as reducing the tumor burden after vaccination in a mouse model.

The shRNA used in the iDR-NC study was used to knock down expression of STAT3, a protein in the JAK-STAT signaling pathway that leads to immunosuppression. This group and others have used RNAi to downregulate immunosuppressive signaling molecules as yet another method of increasing the immunogenicity of tumor microenvironments. In their work, Teo et al. knocked down expression of programmed death ligand-1 (PD-L1), a surface-expressed protein that binds to the programmed death-1 (PD-1) checkpoint molecule on T-cells and leads to hyporesponsiveness [42]. Using cationic PEI to form nanocomplexes with siRNA against PDL1, the authors targeted ovarian cancer cells in vitro by conjugating their polymer with folic acid and showed that knockdown of this checkpoint molecule sensitized cancer cells to T-cell-mediated killing. Heo et al. also used siRNA to knock down an immunosuppressive gene; in their case, they encapsulated siRNA against suppressor of cytokine signaling 1 (SOCS1) into PLGA nanoparticles along with a protein model antigen (ovalbumin) [43] and found that DCs could internalize these particles and improve ovalbumin-specific T-cell activation.

Finally, another interesting avenue of immunotherapy that has found utility clinically and preclinically is the delivery of immunostimulatory cytokines to activate immune cells or relieve immunosuppression, such as in tumor microenvironments. Transfecting tumor cells to express these cytokines would be another way to achieve this effect locally [44-46]. For example, a cationic polypeptide was used in one study to form nanocomplexes with plasmid DNA encoding tumor necrosis factor alpha (TNF-a) [47]. These nanocomplexes could be delivered either intratumorally or systemically and led to dramatically slower tumor growth in a breast tumor model. Other researchers used an amphiphilic, cationic polyphosphazene to deliver a plasmid encoding IL-12 systemically in a murine adenocarcinoma model, resulting in significantly slowed tumor growth [48].

\section{Conclusions}

Engineering immune responses is a complex task, but technologies that can modify cells at the genetic level can lead to new and effective methods of achieving strong results. Nucleic acids can be used to overexpress or knock down specific genes as well as act as adjuvants or danger signals that affect the behavior of immune cells. Cationic polymers can form electrostatic nanocomplexes with nucleic acids to facilitate their entry into cells, while other types of hydrophobic polymers can physically entrap nucleic acids in micro- or nanoparticles for presentation or delivery to cells. APCs are common targets of 
immunoengineering due to their important role in activating or downregulating the immune response, but other cells, like myocytes and cancer cells, are also important targets of immunomodulatory gene delivery as well. Polymer-mediated nucleic acid delivery has progressed vastly over the past decades, and as gene therapy increasingly shows promising results, polymeric nucleic acid delivery for immune stimulation will surely continue to advance as a replacement or complement to the other methods of immunoengineering.

\section{Acknowledgements}

The authors acknowledge the NIH for support (R01EB022148 and R01CA195503). The authors are also thankful for support from the Bloomberg Kimmel Institute for Cancer Immunotherapy and the Research to Prevent Blindness James and Carole Free Catalyst Award.

\section{References}

* Of special interest

** Of outstanding interest

1. Lechardeur D, Verkman AS, Lukacs GL: Intracellular routing of plasmid DNA during non-viral gene transfer. Adv Drug Deliver Rev 2005, 57:755-767.

2. Whitehead KA, Langer R, Anderson DG: Knocking down barriers: advances in siRNA delivery. Nat Rev Drug Discov 2009, 8:129-138. [PubMed: 19180106]

3. Kauffman KJ, Webber MJ, Anderson DG: Materials for non-viral intracellular delivery of messenger RNA therapeutics. J Control Release 2016, 240:227-234. [PubMed: 26718856]

4. Scheiermann J, Klinman DM: Clinical evaluation of $\mathrm{CpG}$ oligonucleotides as adjuvants for vaccines targeting infectious diseases and cancer. Vaccine 2014, 32:6377-6389. [PubMed: 24975812]

5. Schlee M, Hornung V, Hartmann G: siRNA and isRNA: two edges of one sword. Mol Ther 2006, 14:463-470. [PubMed: 16877044]

6. Nguyen DN, Mahon KP, Chikh G, Kim P, Chung H, Vicari AP, Love KT, Goldberg M, Chen S, Krieg AM, Chen J et al.: Lipid-derived nanoparticles for immunostimulatory RNA adjuvant delivery. Proc Natl Acad Sci U S A 2012, 109:E797-803. [PubMed: 22421433]

7. Krasteva PV, Sondermann H: Versatile modes of cellular regulation via cyclic dinucleotides. Nat Chem Biol 2017, 13:350-359. [PubMed: 28328921]

8. Little SR, Kohane DS: Polymers for intracellular delivery of nucleic acids. J Mater Chem 2008, 18:832-841.

9. Pack DW, Hoffman AS, Pun S, Stayton PS: Design and development of polymers for gene delivery. Nat Rev Drug Discov 2005, 4:581-593. [PubMed: 16052241]

10. Yue YA, Jin F, Deng R, Cai JG, Dai ZJ, Lin MCM, Kung HF, Mattebjerg MA, Andresen TL, Wu C: Revisit complexation between DNA and polyethylenimine - Effect of length of free polycationic chains on gene transfection. J Control Release 2011, 152:143-151. [PubMed: 21457737]

11. Green JJ, Langer R, Anderson DG: A combinatorial polymer library approach yields insight into nonviral gene delivery. Acc Chem Res 2008, 41:749-759. [PubMed: 18507402]

12. Ferruti P, Marchisio MA, Duncan R: Poly(amido-amine)s: Biomedical applications. Macromol Rapid Comm 2002, 23:332-355.

13. Kozielski KL, Tzeng SY, Green JJ: A bioreducible linear poly(beta-amino ester) for siRNA delivery. Chem Commun 2013, 49:5319-5321.

14. Nanjwade BK, Bechra HM, Derkar GK, Manvi FV, Nanjwade VK: Dendrimers: Emerging polymers for drug-delivery systems. Eur J Pharm Sci 2009, 38:185-196. [PubMed: 19646528]

15. Leiro V, Garcia JP, Tomas H, Pego AP: The present and the future of degradable dendrimers and derivatives in theranostics. Bioconjug Chem 2015, 26:1182-1197. [PubMed: 25826129] 
16. Mao SR, Sun W, Kissel T: Chitosan-based formulations for delivery of DNA and siRNA. Adv Drug Deliver Rev 2010, 62:12-27.

17. Walter E, Moelling K, Pavlovic J, Merkle HP: Microencapsulation of DNA using poly(DL-lactideco-glycolide): stability issues and release characteristics. J Control Release 1999, 61:361-374. [PubMed: 10477808]

18. Woodrow KA, Cu Y, Booth CJ, Saucier-Sawyer JK, Wood MJ, Saltzman WM: Intravaginal gene silencing using biodegradable polymer nanoparticles densely loaded with small-interfering RNA. Nat Mater 2009, 8:526-533. [PubMed: 19404239]

19**. Smith TT, Stephan SB, Moffett HF, McKnight LE, Ji WH, Reiman D, Bonagofski E, Wohlfahrt ME, Pillai SPS, Stephan MT: In situ programming of leukaemia-specific T cells using synthetic DNA nanocarriers. Nat Nanotechnol 2017, 12:813-820.

This report describes a system that delivers DNA with high efficacy and specificity to T-cells for an antigen-specific anti-tumor response.

[PubMed: 28416815]

20. Christensen LV, Chang CW, Kim WJ, Kim SW, Zhong ZY, Lin C, Engbersen JFJ, Feijen J: Reducible poly(amido ethylenimine)s designed for triggered intracellular gene delivery. Bioconjugate Chem 2006, 17:1233-1240.

21. Banchereau J, Briere F, Caux C, Davoust J, Lebecque S, Liu YT, Pulendran B, Palucka K: Immunobiology of dendritic cells. Annu Rev Immunol 2000, 18:767-811. [PubMed: 10837075]

22. Sousa CR: Dendritic cells in a mature age. Nat Rev Immunol 2006, 6:476-483. [PubMed: 16691244]

23. Huppa JB, Davis MM: T-cell-antigen recognition and the immunological synapse. Nat Rev Immunol 2003, 3:973-983. [PubMed: 14647479]

24. Guermonprez P, Valladeau J, Zitvogel L, Thery C, Amigorena S: Antigen presentation and T cell stimulation by dendritic cells. Annu Rev Immunol 2002, 20:621-667. [PubMed: 11861614]

25. Tanaka A, Sakaguchi S: Regulatory T cells in cancer immunotherapy. Cell Res 2017, 27:109-118. [PubMed: 27995907]

26. Kaech SM, Wherry EJ, Ahmed R: Effector and memory T-cell differentiation: Implications for vaccine development. Nat Rev Immunol 2002, 2:251-262. [PubMed: 12001996]

27. Orenstein WA, Ahmed R: Simply put: Vaccination saves lives. Proc Natl Acad Sci U S A 2017, 114:4031-4033. [PubMed: 28396427]

28. Coffman RL, Sher A, Seder RA: Vaccine Adjuvants: Putting Innate Immunity to Work. Immunity 2010, 33:492-503. [PubMed: 21029960]

29. Klinman DM, Klaschik S, Tross D, Shirota H, Steinhagen F: FDA guidance on prophylactic DNA vaccines: Analysis and recommendations. Vaccine 2010, 28:2801-2805. [PubMed: 19941989]

30. Jilek S, Ulrich M, Merkle HP, Walter E: Composition and surface charge of DNAloaded microparticles determine maturation and cytokine secretion in human dendritic cells. Pharm Res 2004, 21:1240-1247. [PubMed: 15290866]

31. Zhou X, Liu B, Yu X, Zha X, Zhang X, Chen Y, Wang X, Jin Y, Wu Y, Chen Y, Shan Y et al.: Controlled release of PEI/DNA complexes from mannose-bearing chitosan microspheres as a potent delivery system to enhance immune response to HBV DNA vaccine. J Control Release 2007, 121:200-207. [PubMed: 17630014]

32. Chen YZ, Yao XL, Ruan GX, Zhao QQ, Tang GP, Tabata Y, Gao JQ: Gene-carried chitosan-linked polyethylenimine induced high gene transfection efficiency on dendritic cells. Biotechnol Appl Biochem 2012, 59:346-352. [PubMed: 23586911]

33**. Kim NW, Lee MS, Kim KR, Lee JE, Lee K, Park JS, Matsumoto Y, Jo DG, Lee H, Lee DS, Jeong JH: Polyplex-releasing microneedles for enhanced cutaneous delivery of DNA vaccine. $\mathbf{J}$ Control Release 2014, 179:11-17.

This report describes a device engineered to deliver DNA as a vaccine while overcoming barriers to application and immunogenicity.

[PubMed: 24462900] 
34. DeMuth PC, Min YJ, Huang B, Kramer JA, Miller AD, Barouch DH, Hammond PT, Irvine DJ: Polymer multilayer tattooing for enhanced DNA vaccination. Nature Materials 2013, 12:367-376. [PubMed: 23353628]

35. Shirota H, Tross D, Klinman DM: CpG oligonucleotides as cancer vaccine adjuvants. Vaccines 2015, 3:390-407. [PubMed: 26343193]

36. Hafner AM, Corthesy B, Merkle HP: Particulate formulations for the delivery of poly(I:C) as vaccine adjuvant. Adv Drug Deliver Rev 2013, 65:1386-1399.

37. Banchereau J, Palucka AK: Dendritic cells as therapeutic vaccines against cancer. Nat Rev Immunol 2005, 5:296-306. [PubMed: 15803149]

38. Corrales L, Glickman LH, McWhirter SM, Kanne DB, Sivick KE, Katibah GE, Woo SR, Lemmens E, Banda T, Leong JJ, Metchette K et al.: Direct activation of STING in the tumor microenvironment leads to potent and systemic tumor regression and immunity. Cell Rep 2015, 11:1018-1030. [PubMed: 25959818]

39*. Wilson DR, Sen R, Sunshine JC, Pardoll DM, Green JJ, Kim YJ: Biodegradable STING agonist nanoparticles for enhanced cancer immunotherapy. Nanomed-Nanotechnol 2018, 14:237-246.

This report showed significant anti-tumor efficacy of CDNs when comiplexed with PBAEs and injected intratumorally.

40*. Leach DG, Dharmaraj N, Piotrowski SL, Lopez-Silva TL, Lei YL, Sikora AG, Young S, Hartgerink JD: STINGel: Controlled release of a cyclic dinucleotide for enhanced cancer immunotherapy. Biomaterials 2018, 163:67-75.

The described delivery vehicle combines immunostimulatory CDNs with a controlled release system for sustained immunostimulatory effect.

[PubMed: 29454236]

41*. Zhu GZ, Mei L, Vishwasrao HD, Jacobson O, Wang ZT, Liu YJ, Yung BC, Fu X, Jin A, Niu G, Wang $\mathrm{Q}$ et al.: Intertwining DNA-RNA nanocapsules loaded with tumor neoantigens as synergistic nanovaccines for cancer immunotherapy. Nat Commun 2017, 8

This report describes an innovative design that combines multiple nucleic acids with different immunological effects, including direct knockdown of gene expression and activation of innate immunity.

42. Teo PY, Yang C, Whilding LM, Parente-Pereira AC, Maher J, George AJT, Hedrick JL, Yang YY, Ghaem-Maghami S: Ovarian cancer immunotherapy using PD-L1 siRNA targeted delivery from folic acid-functionalized polyethylenimine: Strategies to enhance T cell killing. Adv Healthc Mater 2015, 4:1180-1189. [PubMed: 25866054]

43. Heo MB, Cho MY, Lim YT: Polymer nanoparticles for enhanced immune response: Combined delivery of tumor antigen and small interference RNA for immunosuppressive gene to dendritic cells. Acta Biomater 2014, 10:2169-2176. [PubMed: 24394635]

44. Maheshwari A, Mahato RI, McGregor J, Han SO, Samlowski WE, Park JS, Kim SW: Soluble biodegradable polymer-based cytokine gene delivery for cancer treatment. Mol Ther 2000, 2:121130. [PubMed: 10947939]

45. Kim TH, Jin H, Kim HW, Cho MH, Cho CS: Mannosylated chitosan nanoparticlebased cytokine gene therapy suppressed cancer growth in BALB/c mice bearing CT26 carcinoma cells. Mol Cancer Ther 2006, 5:1723-1732. [PubMed: 16891458]

46. Fewell JG, Matar MM, Rice JS, Brunhoeber E, Slobodkin G, Pence C, Worker M, Lewis DH, Anwer K: Treatment of disseminated ovarian cancer using nonviral interleukin12 gene therapy delivered intraperitoneally. J Gene Med 2009, 11:718-728. [PubMed: 19507172]

47. Shukla V, Dalela M, Vij M, Weichselbaum R, Kharbanda S, Ganguli M, Kufe D, Singh H: Systemic delivery of the tumor necrosis factor gene to tumors by a novel dual DNA-nanocomplex in a nanoparticle system. Nanomed-Nanotechnol 2017, 13:1833-1839.

48. Gao MH, Zhu XM, Wu LP, Qiu LY: Cationic Polyphosphazene Vesicles for Cancer Immunotherapy by Efficient in Vivo Cytokine IL-12 Plasmid Delivery. Biomacromolecules 2016, 17:2199-2209. [PubMed: 27192398] 


\section{Highlights}

- $\quad$ Polymers can be formulated with nucleic acids to enhance their delivery to cells

- DNA or mRNA encoding antigens can be used as nucleic acid-based vaccines

- Nucleic acids can code for immunostimulatory genes or downregulate inhibitory genes

- Immunostimulatory nucleic acids can activate innate immunity as adjuvants 

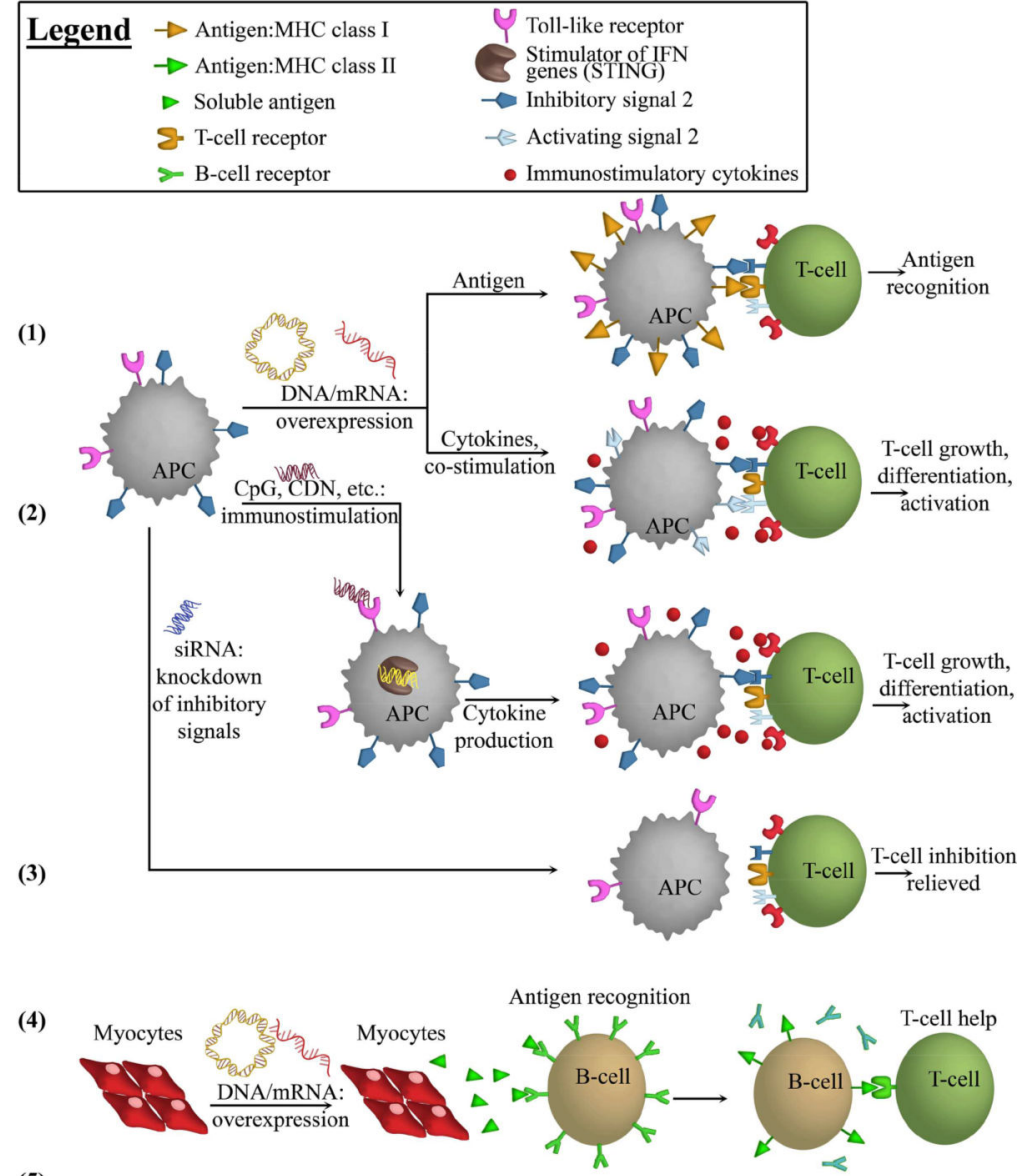

(5) Tumor
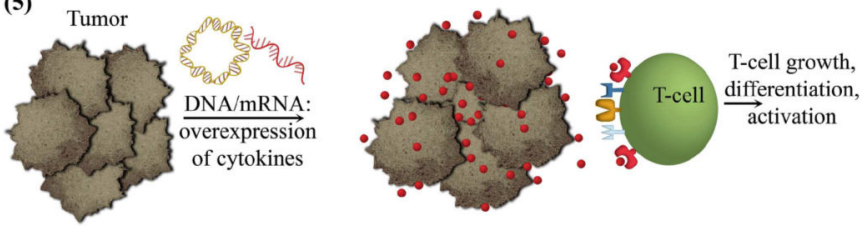

Figure 1.

APCs can be transfected with (1) DNA or mRNA to express antigen, leading to antigen recognition by T-cells, or cytokines or co-stimulatory molecules, leading to T-cell growth, differentiation, and activation; (2) immunostimulatory nucleic acids that bind to innate immune proteins like TLRs or STING, leading to expression of activating signals; or (3) siRNA or shRNA, knocking down expression of inhibitory signals. (4) Myocytes can be transfected by DNA or mRNA to express antigens that can be recognized by B-cells or be taken up by APCs for recognition by B-cells. (5) Tumor cells can also be transfected with DNA or mRNA to express immunostimulatory cytokines that lead to T-cell activation. 

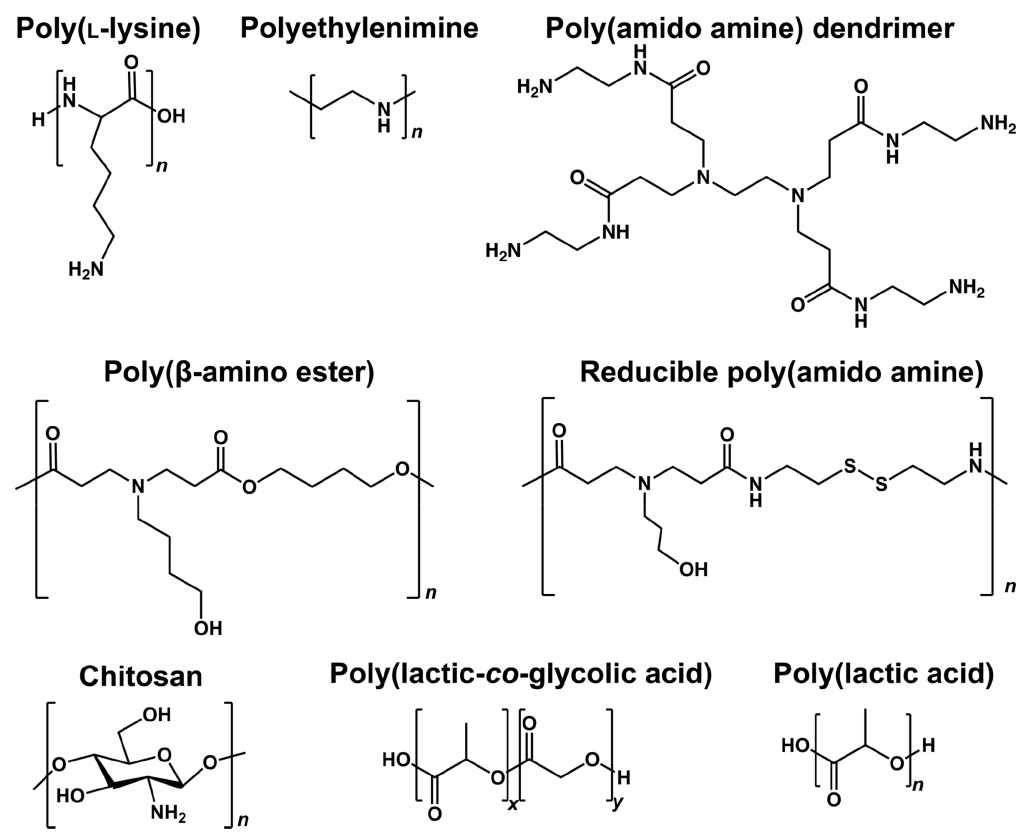

Reducible poly(amido amine)<smiles>CNCCSSCCNC(=O)CCN(CCO)CCC(C)=O</smiles>

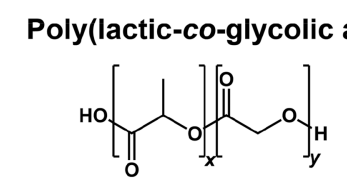

Poly(lactic acid)

Figure 2.

A wide range of polymers can be used for nucleic acid delivery. Commonly used structures often contain features such as cationic charge and degradable linkages. 


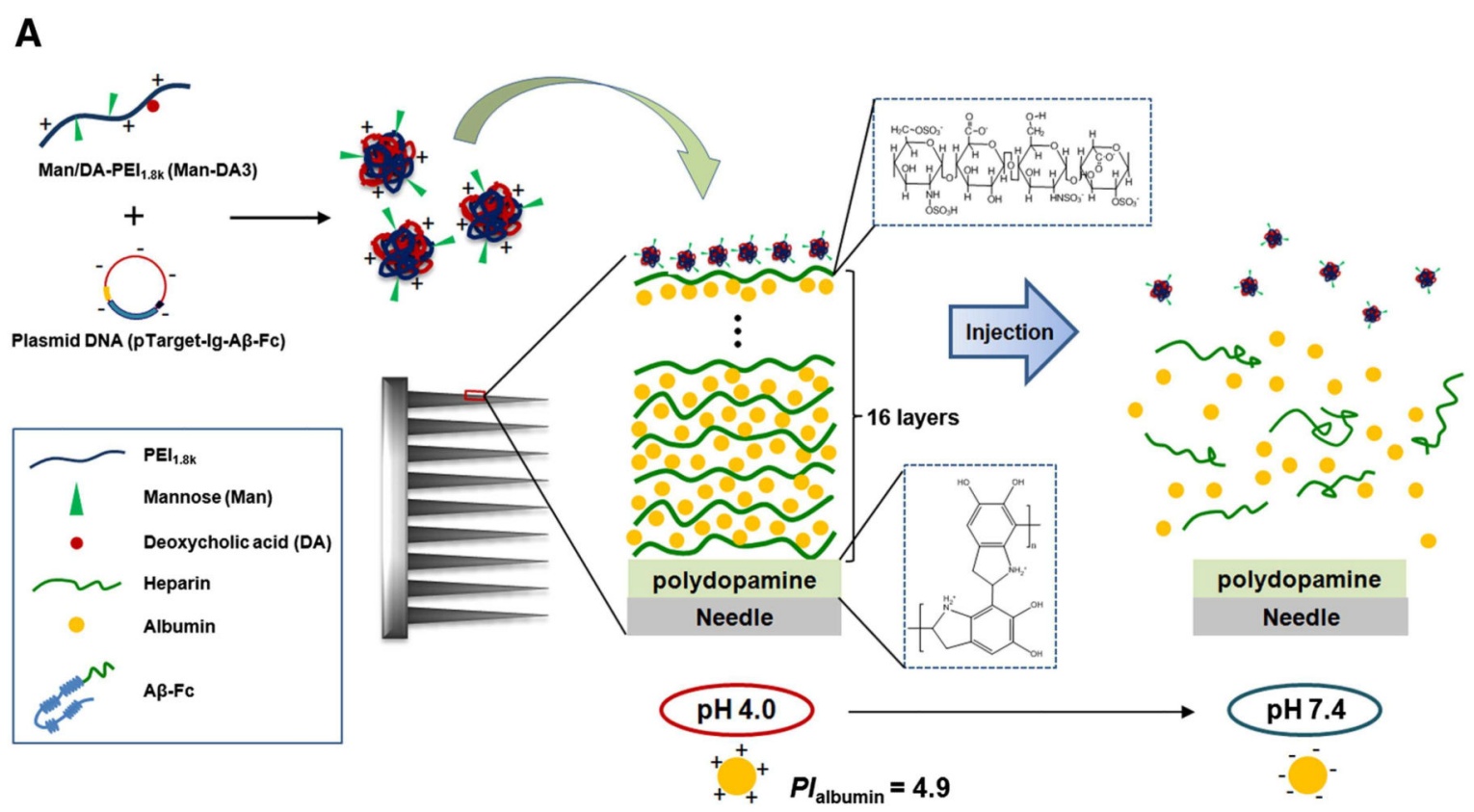

B

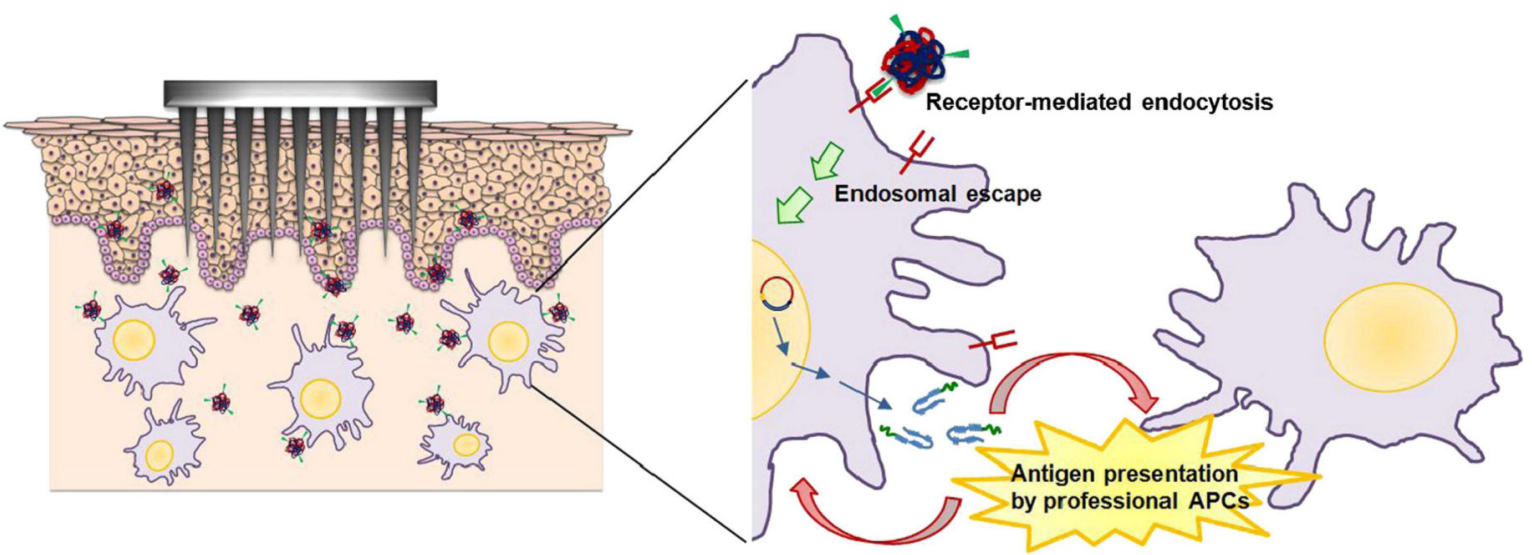

Figure 3.

Plasmid DNA encoding the $a \beta$ antigen was loaded into microneedles with the cationic polymer Man-DA3. Application to skin allowed immune cells at that site to take up the DNA/polymer polyplexes and express and process the antigen for presentation to lymphocytes. Reprinted from Journal of Controlled Release, 179, Kim et al., Polyplexreleasing microneedles for enhanced cutaneous delivery of DNA vaccine, pg. 11-17, Copyright (2014), with permission from Elsevier [33]. 

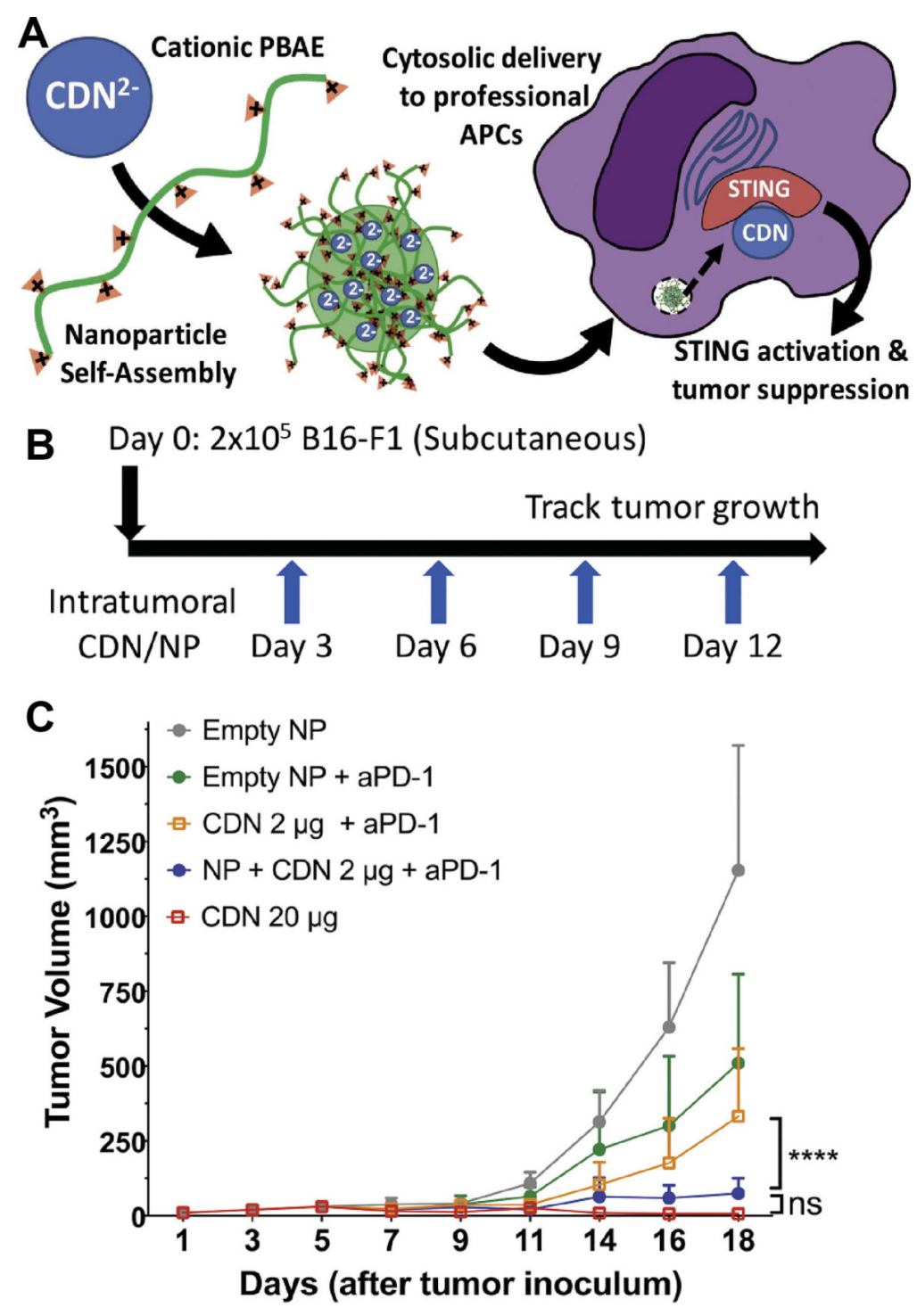

Figure 4.

[39] Schematic of CDN complexation with cationic PBAE to form nanoparticles that can enter APCs and activate STING (A). Intratumoral injection of CDN/PBAE nanoparticles (B) leads to slowed tumor growth in an immunocompetent mouse model of melanoma (C). Reprinted from Nanomedicine: Nanotechnology, Biology and Medicine, 14(2), Wilson et al., Biodegradable STING agonist nanoparticles for enhanced cancer immunotherapy, pg. 237246, Copyright (2018), with permission from Elsevier [39]. 